\title{
1 Documentation, process and implementation of advance care planning in the 2 pediatric population: A scoping review protocol.
}

3

4 Colleen Pawliuk, MLIS, Department of Pediatrics, University of British Columbia/BC Children's 5
Hospital, Vancouver Canada

Nadine Lusney, BScN, RN, MN, CHPCN(C), Canuck Place Children's Hospice, Vancouver, Canada

Camara van Breemen, NP, MN, CHPCN(C), Canuck Place Children's Hospice, Vancouver, Canada; Two World Cancer Collaboration Foundation, Kelowna, Canada; Faculty of Medicine, University of British Columbia, Vancouver, Canada

Zahra Hussein, MPH, MBA, ChildHealth BC, Vancouver, Canada

\section{Abstract}

Objective: To explore the literature surrounding pediatric ACP process, documenting and implementing the documentation processes in hopes to inform and support content experts in the creation of a pediatric provincial document for ACP.

Introduction: The number of children with serious illness/medical complexity in this province who require $A C P$ and documentation of medical intervention is estimated to be $>500$ or possibly more. An ongoing data analysis suggests that the number may exceed 1,000 children, greater than the number of children and families consulted to the $\mathrm{CPCH}$ program. Currently there is no provincial document for pediatric patients such as the BC Medical Orders for Scope of Treatment form (MOST) as there is in adult care.

Inclusion criteria: The review will include studies that focus on documentation, process or implementation in pediatric advance care planning. Child refers to prenatal, neonate, child and youth population (0-19 years), but may also extend into young adulthood in some contexts. These children are defined as at-risk for sudden death and/or expected death prior to reaching adulthood. 
27 Methods: We will search MEDLINE (Ovid), Embase (Ovid), CINAHL (EBSCOhost), Web of Science Core 28 Collection and Google Scholar, as well as sources of unpublished studies and grey literature.

29 Identified studies will be screened against the eligibility criteria. This review will only consider studies published in English and no date limits will be placed on the search. Relevant data will be extracted from eligible studies and the extracted data will presented in narrative and tabular/charted formats.

33 Keywords: advance care planning, documentation, pediatric 


\section{Introduction}

Currently, there is an absence of overarching provincial guidance and documentation of Goals of Care for children with serious illness medical complexity (i.e., life-threatening/ life-limiting illnesses) requiring Advance Care Planning ( $A C P$ ) in British Columbia (BC), Canada. Child refers to prenatal, neonate, child and youth population (0-19 years), but may also extend into young adulthood in some contexts. These children are defined as at-risk for sudden death and/or expected death prior to reaching adulthood. Pediatric ACP is a process that seeks to explore values, beliefs and wishes to support informed healthcare decision-making and guide future healthcare decisions within a fit for the child/family ${ }^{1-3}$. It recognizes the role, voice and values of the child and the relationship of the child within a family context. ACP requires a high degree of communication competency and can lead to better outcomes for families and systems-research has shown decreased number of emergency visits; enhanced ability of parents to plan for most ideal location of care; decreased parental decisional regret and child suffering at end of life ${ }^{4-6}$. Pediatric clinicians face additional barriers to those in adult palliative care such as caring for children too young to express their wishes, collaborating with parents as surrogate decision-makers, treating rare diseases for which prognostication is particularly difficult, and interacting with multiple healthcare providers and teams with differing communication competencies ${ }^{4-6}$. ACP can also reduce healthcare provider stress and moral distress by providing clarity regarding family wishes during crises ${ }^{7}$. In pediatrics, this also includes considering the child's ability to understand, participate and make specific decisions about their care. Pediatric ACP (pACP) involves the concept of parallel planning - planning for the life of the child while also planning for deterioration/death to allow for the child's full potential ${ }^{8}$. This primes the mobilization of services and healthcare professionals when necessary. An Advance Care Plan may at times look like an anticipatory care plan - laying out actions to be taken if or when a child's illness is unstable or deteriorates or they develop life-threatening complications due to their illness. Having a specific overarching provincial guideline for initiating and documenting complex conversations surrounding $A C P$, goals of care $(G O C)$ and levels of interventions specific to pediatrics serves to enhance clinicians' confidence, maintain a consistent message from clinician to clinician, and addresses family's potential concerns ${ }^{7,9}$.

Canuck Place Children's Hospice ( $\mathrm{CPCH}$ ) is the provincial pediatric palliative care program in $\mathrm{BC}$. Pediatric palliative care is a model of care, appropriate at any stage of the illness, and can be provided together with disease directed treatment. It involves integrated care directed at the physical, emotional, spiritual, and social needs of the child and family, delivered by an integrated 
and multidisciplinary team ${ }^{10}$. CPCH currently supports hundreds of families across BC with pain and symptom control, ACP and enhanced communication, family support and care coordination. The number of children with serious illness/medical complexity in this province who require ACP and documentation of medical intervention is estimated to be $>500$ or possibly more. An ongoing data analysis suggests that the number may exceed 1,000 children, greater than the number of children and families consulted to the $\mathrm{CPCH}$ program. Documenting GOC occurs in a variety of settings that are often not well linked thus creating tension and difficulty for parents who are attempting to communicate their wishes. It also leads to confusion for healthcare providers about the type of care to provide as it is unclear as to how or where to document in order to promote continuity of care and shared understanding. Currently there is no provincial document for pediatric patients such as the BC Medical Orders for Scope of Treatment form (MOST) as there is in adult care. A recent case of a clinician within a $\mathrm{BC}$ health authority not contacting/consulting the $\mathrm{CPCH}$ program at the time has highlighted the gap in ACP as local providers were unsure how to have the ACP conversation with the family of a child, and then how best to document the limits of medical intervention. The number of children requiring ACP in B.C. is relatively low; however, the impact on the family and healthcare provider could be significant ${ }^{7,9}$.

Recently, the BC Center for Palliative Care, in collaboration with Palliative Care content experts across the province, engaged in the process of updating the adult MOST form through a provincial working group, FLICS. Expected completion date for the project is December 2021. BC Center for Palliative Care was established in 2013 to support the province's end-of-life care action plan. Due to the constraints of the Covid-19 pandemic, implementing the updated MOST form will be the responsibility of each health authority. Currently, the Ministry is not involved in the project. CPCH and ChildHealth $B C$ are interested in exploring the literature surrounding pediatric ACP process, documenting and implementing the documentation processes in hopes to inform and support content experts in the creation of a pediatric provincial document for ACP. ChildHealth BC is a provincial health network whose Steering Committee brings together child health leaders to enable the most innovative, novel and sustainable service for children's health.

A preliminary search of PROSPERO, MEDLINE and the Cochrane Database of Systematic Reviews was conducted and no current or in-progress scoping reviews or systematic reviews on the topic were identified.

The objective is to conduct a scoping review of research literature and grey literature related to the 
topic of pACP (process, documentation, implementation). The scoping review will take into consideration the literature review process undertaken by the FLICS project; however, the pediatric scoping review search will likely be more expansive since the literature is likely to be limited. In addition, the scoping review will include an environmental scan of current pediatric ACP documents, and process and practices across BC Health Authorities, Canada and possibly beyond as per the lit review and environmental process completed by FLICS for the adult population. This information, along with collaboration with key content experts across the province, will compose the foundations for a provincial pediatric ACP document and process.

\section{Review question(s)}

What practices exist for documentation, process and implementation of advance care planning in the pediatric population?

\section{Inclusion criteria}

This review will consider studies that include participants who are 0-19 years old. Considerations will be made to include studies that include a mixed population of pediatric participants and young adults or studies focused on young adults as the age range for young adults versus pediatric might be different in other countries.

\section{Concept}

This review will consider studies that explore advance care planning process, documentation and the implementation of advance care planning documentation.

\section{Context}

This review will consider studies from all healthcare settings, including in-home care, hospital and community care, in all geographic locations.

\section{Types of sources}

This scoping review will consider quantitative, qualitative, and mixed methods study designs for inclusion. In addition, systematic reviews and text and opinion papers will be considered for inclusion in the proposed scoping review. We will also consider ACP documents, practices and processes identified in our environmental scan. 


\section{Methods}

The proposed scoping review will be conducted in accordance with the JBI methodology for scoping reviews. ${ }^{11}$

\section{Search strategy}

The search strategy will aim to locate both published and unpublished primary studies, reviews, and text and opinion papers. An initial limited search of MEDLINE (Ovid)and CINAHL (EBSCOhost) was undertaken to identify articles on the topic (see Appendix I). The text words contained in the titles and abstracts of relevant articles, and the index terms used to describe the articles, will be used to develop a full search strategy for MEDLINE (Ovid). The search strategy, including all identified keywords and index terms, will be adapted for each included information source. The reference lists of articles included in the review will be screened for additional papers.

Articles published in English will be included due to a lack of resources to translate non-English articles. Articles published from database inception to the present will be included.

The databases to be searched include MEDLINE (Ovid), Embase (Ovid), CINAHL (EBSCOhost), Web of Science Core Collection and Google Scholar. Sources of unpublished studies and grey literature to be searched include:

- Theses and Dissertations:

- ProQuest Theses and Dissertations Global

○ Networked Digital Library of Theses and Dissertations (NDLTD)

- Conference Proceedings:

- Papers First (via Worldcat First Search)

- Proceedings (via Worldcat First Search)

- Canadian Organizational Websites:

- Speak Up Canada (https://www.advancecareplanning.ca/)

- Speakup Canada pediatric resources (https://www.advancecareplanning.ca/national-community-of-practice-for-advancecare-planning-educators/document-library/pediatric-advance-care-planning/)

- BC Center for Palliative Care (https://bc-cpc.ca/all-resources/individuals/acp/; https://bc-cpc.ca/all-resources/individuals/acp/acp-resources/)

○ Healthlink BC (https://www.healthlinkbc.ca/) 
○ Government BC- My Voice

(https://www.health.gov.bc.ca/library/publications/year/2013/MyVoiceAdvanceCarePlanningGuide.pdf)

○ Interior health (https://www.interiorhealth.ca/sites/Partners/palliative/Pages/Clinical-PracticeSupports.aspx)

- Vancouver Island Health Authority (https://www.islandhealth.ca/ourservices/advance-care-planning/advance-care-planning)

○ Fraser Health (https://www.fraserhealth.ca/health-topics-a-to-z/advance-careplanning\#.YJ1t6KhKhdg)

- PHSA (http://www.phsa.ca/health-info/advance-care-planning)

- Canadian Virtual Hospice (https://www.virtualhospice.ca/en_US/Main+Site+Navigation/Home.aspx)

- Ministry of Children and Family Development (https://www2.gov.bc.ca/gov/content/governments/organizationalstructure/ministries-organizations/ministries/children-and-family-development)

○ Healthlink (https://www.healthlinkbc.ca/health-feature/advance-care-planning)

- Other PPC programs across Canada or key PPC programs, including Sick Kids Hospital, Rogers House, Flames House, IWK hospital, Emily's House, etc.

- Search Engine:

- Google searches targeting Pediatric Palliative Care Programs and Hospices in Australia, US, New Zealand, UK

\section{Study/Source of evidence selection}

Following the search, all identified records will be collated and uploaded into Covidence and duplicates removed. Following a pilot test, titles and abstracts will then be screened by two independent reviewers for assessment against the inclusion criteria for the review. The full text of selected citations will be assessed in detail against the inclusion criteria by two independent reviewers. Reasons for exclusion of full-text papers that do not meet the inclusion criteria will be recorded and reported in the scoping review. Any disagreements that arise between the reviewers at each stage of the selection process will be resolved through discussion or with a third reviewer. The results of the search will be reported in full in the final scoping review and presented in a 
Preferred Reporting Items for Systematic Reviews and Meta-analyses for Scoping Reviews (PRISMAScR) flow diagram. ${ }^{12}$

\section{Data extraction}

Data will be extracted from papers included in the scoping review by two independent reviewers using a data extraction tool developed by the reviewers. The minimum data to be extracted are:

- Number of participants

- Age range

- Country

- Study design

- Person who conducts the ACP conversation (e.g. GP, specialist, paramedic)

- Characteristics of ACP forms

The draft data extraction tool will be modified and revised as necessary during the process of extracting data from each included paper. Modifications will be detailed in the full scoping review. Any disagreements that arise between the reviewers will be resolved through discussion or with a third reviewer. Authors of papers will be contacted to request missing or additional data, where required.

\section{Data analysis and presentation}

The extracted data will be presented in diagrammatic or tabular form in a manner that aligns with the objective of this scoping review. A narrative summary will accompany the tabulated or charted results, and will describe how the results relate to the reviews objective and question.

\section{Funding}

Funding for this scoping review is provided by Canuck Place Children's Hospice.

\section{Conflicts of interest}

The authors declare no conflict of interest.

\section{References}

1. Downing J, Ling J, Benini F, et al. Core competencies for Education in Paediatric Palliative Care. Report of the EAPC Children's Palliative Care Education Taskforce, November 2013. Milan: European Association for Palliative Care (EAPC) 
2. Together for Short Lives. A core care pathway for children with life-limiting and lifethreatening condition, $3^{\text {rd }}$ ed. Bristol: Together for Short Lives, 2013.

3. Pediatric Hospice Palliative Care Guiding Principles and Norms of Practice [internet] [cited 2018 May 25] Ottawa (ON): Canadian Hospice Palliative Care Association.; 2006. Available from: http://www.chpca.net/media/7841/Pediatric Norms of Practice March 312006 English. pdf

4. Kassam A, Skiadaresis J, Alexander S, Wolfe J. Differences in end-of-life communication for children with advanced cancer who were referred to a palliative care team. Pediatr Blood Cancer 2015;62:1409-13.

5. Snaman JM, Kaye EC, Lu JJ, Sykes A, Baker JN._Palliative Care Involvement is Associated with Less Intensive End-of-life Care in Adolescents and Young Adult Oncology Patients. J Palliat Med. 2017;20:509-16.

6. Ananth P, Melvin P, Berry JG, Wolfe J. Trends in Hospital Utilization and Costs among Pediatric Palliative Care Recipients. J Palliat Med. 2017;20:946-53.

7. Lotz JD, Jox RJ, Borasio GD, Fuhrer M. Pediatric advance care planning from the perspective of health care professions: a qualitative interview study. Palliat Med. 2015;29:212-22.

8. Sidgwick, Fraser, Fortune, $P, \&$ McCulloch. Parallel Planning and the pediatric intensive care patient. BMJ 2019: 104 (10).

9. Lotz, JD, Daxer M, Jox RJ, Borasio GD, Fuhrer M. "Hope for the best, prepare for the worst": A qualitative interview study on parents' needs and fears in pediatric advance care planning. Palliat Med. 2017;31:764-71.

10. Siden, H. (2018). Pediatric Palliative Care for Non-Malignant Disease. Children (5) 28, 1-9.

11. Tufanaru C, Munn Z, Aromataris E, Campbell J, Hopp L. Chapter 3: Systematic reviews of effectiveness. In: Aromataris E, Munn Z (Editors). JBI Manual for Evidence Synthesis [Internet]. Adelaide: JBI; 2020 [cited 2021 AUG 31]. Available from:https://synthesismanual.jbi.global.

12. Page MJ, McKenzie JE, Bossuyt PM, et al. The PRISMA 2020 statement: an updated guideline for reporting systematic reviews. BMJ. 2021;372:n71. 


\section{Appendix I: MEDLINE Search strategy}

Ovid MEDLINE(R) and Epub Ahead of Print, In-Process, In-Data-Review \& Other NonIndexed Citations, Daily and Versions(R) <1946 to September 01, 2021>

\begin{tabular}{|c|c|c|}
\hline$\#$ & Query & \begin{tabular}{|l} 
Results \\
from 2 \\
Sep 2021 \\
\end{tabular} \\
\hline 1 & advance care planning/ or advance directives/ or living wills/ & 10,186 \\
\hline 2 & Advance Directive Adherence/ & 529 \\
\hline 3 & advance? care planning.tw,kf. & 4,220 \\
\hline 4 & (advance? adj2 directive?).tw,kw. & 4,556 \\
\hline 5 & Resuscitation Orders/ & 4,027 \\
\hline 6 & resuscitation order?.tw,kf. & 300 \\
\hline 7 & "do not resuscitate".tw,kf. & 2,321 \\
\hline 8 & "do not attempt resuscitation".tw,kf. & 250 \\
\hline 9 & $\mathrm{or} / 1-8$ & 17,273 \\
\hline 10 & Documentation/ & 18,333 \\
\hline 11 & document*.tw,kf. & 433,332 \\
\hline 12 & (create or creation).tw,kf. & 209,345 \\
\hline 13 & develop*.tw,kf. & $4,770,423$ \\
\hline 14 & form?.tw,kf. & $1,418,215$ \\
\hline 15 i & implement*.tw,kf. & 567,094 \\
\hline 16 & or/10-15 & $6,654,282$ \\
\hline 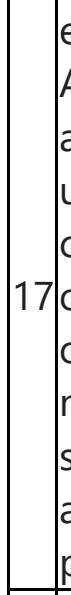 & 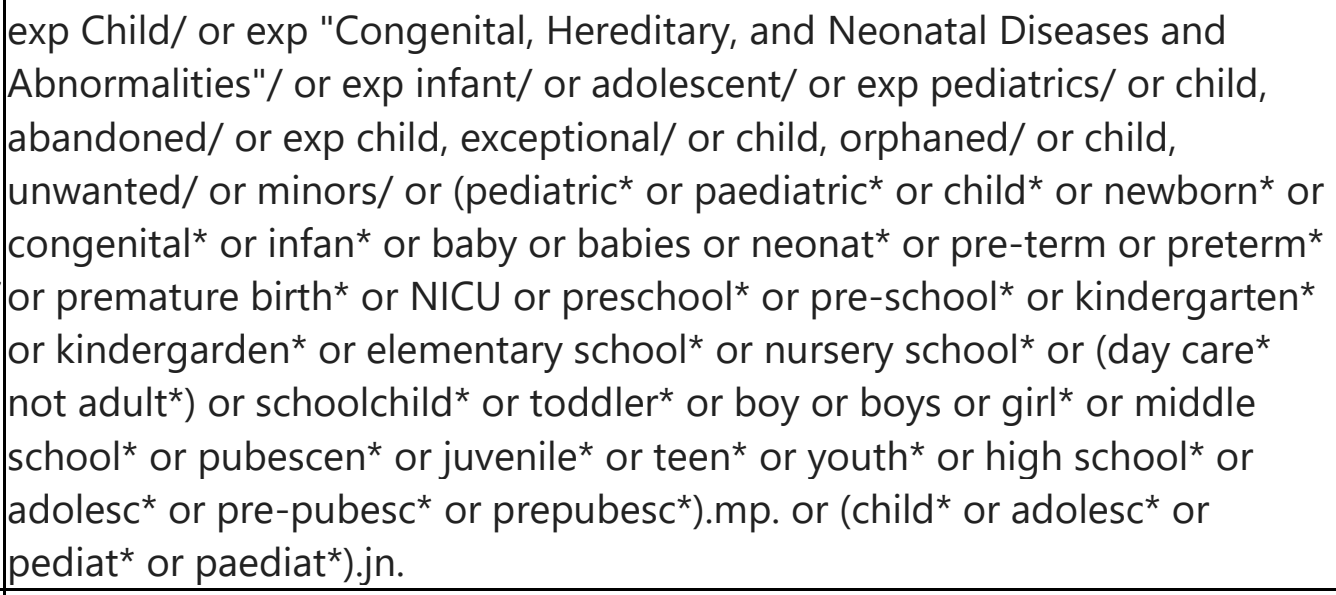 & $5,348,615$ \\
\hline 189 & 9 and 16 and 17 & 580 \\
\hline 19 & limit 18 to english language & 549 \\
\hline
\end{tabular}

